\title{
Two-Dimensional (2D-J) NMR Spectroscopy for Analysis of Isomers and Heterocouplings
}

\author{
Reinhard Benn* \\ Max-Planck-Institut für Kohlenforschung, Kaiser-Wilhelm-Platz 1, \\ D-4330 Mülheim an der Ruhr, West Germany \\ Werner Riemer \\ Institut für Strahlenchemie im Max-Planck-Institut für Kohlenforschung, Stiftstraße 34-36, \\ D-4330 Mülheim an der Ruhr, West Germany
}

Z. Naturforsch. 36 b, 488-491 (1981); received November 27, 1980

2-Dimensional NMR, Stereoisomers, Heterocouplings

The use of two-dimensional NMR spectroscopy (2D-J) for the identification of isomers and heterocouplings is demonstrated. The routine application of this new method to practical NMR problems is discussed.

\section{Introduction}

NMR spectra can be recorded by displaying the intensity of absorption not only as a function of one frequency but also as a function of a second or third variable. This original idea has been suggested by Jeener in 1971 [1] and since then the basic principles and some pioneer experiments of multidimensional NMR spectroscopy have been developed by several groups [2, 3]. In particular, it has become possible to record two dimensional NMR spectra (a twodimensional NMR spectrum is a plot of intensity versus two frequency variables). The basic principles of this technique (2D-NMR) have been worked out by Ernst [4] and Freeman [5]. Recently it has been shown that $2 \mathrm{D}-\mathrm{NMR}$ is suitable for the study of a variety of phenomena, such as multiquantum transitions [6], ${ }^{13} \mathrm{C}-{ }^{13} \mathrm{C}$ coupling constants in natural abundance spectra [7] or exchange phenomena [8]. A special case of $2 \mathrm{D}$-spectroscopy is $2 \mathrm{D}-\mathrm{J}$ resolved spectroscopy $[4,5]$. For weakly coupled spins in $2 \mathrm{D}-\mathrm{J}$ NMR the chemical shift of a nucleus is displayed in one dimension and the coupling of the nuclei with each other (or with an other magnetically active nucleus) is displayed in the second frequency dimension.

The basis of a $2 \mathrm{D}-\mathrm{J}$ resolved spectrum is a spin echo experiment [9] consisting of a $90^{\circ}-t_{1} / 2-$ $180^{\circ}-t_{1} / 2-F I D\left(t_{2}\right)$ pulse sequence. The spin echoes are modulated by homonuclear couplings since the coupled nuclei experience the effects of the

* Reprint requests to Dr. R. Benn.

0340-5087/81/0400-0488/\$01.00/0 refocusing pulse. If the period $t_{1}$ is varied the phase error becomes a phase modulation and this can be transformed into an amplitude modulation. The second half of the spin echo $F I D\left(t_{2}\right)$ resembles a free induction decay (the FID is a function of $t_{2}$ ). The detected signal therefore is a function of $t_{1}$ and $t_{2}$. It is transformed first with respect to $t_{2}$ and then with respect to $t_{1}$ to produce the two dimensional spectrum $\mathrm{F}\left(\omega_{1}, \omega_{2}\right)$. The $\omega_{2}$ frequency dimension is that of conventional NMR (i.e. the chemical shift axis). The homocouplings appear in $\omega_{1}$ frequency dimension. Thus orthogonal projection in $\omega_{1}$ direction on the $\omega_{2}$ dimension results in a type of "broad band ${ }^{1} \mathrm{H}$ decoupled" proton spectrum consisting of singlets (in the absence of heterocouplings) whereas projection in $\omega_{2}$ direction on the $\omega_{1}$ axis yields the homonuclear multiplet patterns of the corresponding resonances.

Although this new technique has been known for some years its application to practical NMR is still rather limited. The aim of this paper is to show the use of 2D-J NMR spectroscopy in routine NMR analysis: in particular the utility of $2 \mathrm{D}-\mathrm{J}$ NMR in the identification of (stereo)isomers and heterocouplings will be emphazised.

\section{Experimental}

All spectra were recorded on a Bruker WH 400 spectrometer, equipped with an Aspect 2000 computer with a $80 \mathrm{~K}$ memory and a double drive high density disk. The $2 \mathrm{D}-\mathrm{J}$ resolved spectra were recorded with a development version of the FT-NMR-2D program. The number of data points in the $t_{1}\left(\omega_{1}\right)$ dimension was 256 and in the $t_{2}\left(\omega_{2}\right)$ dimension 4096; the corresponding spectral width was $\pm 17.816 \mathrm{~Hz}$ and $570.125 \mathrm{~Hz}$ leading to a digital 
resolution of $0.139 \mathrm{~Hz}$ in the $t_{1}\left(\omega_{1}\right)$ and $0.278 \mathrm{~Hz}$ in the $t_{2}\left(\omega_{2}\right)$ dimension. The $90^{\circ}$ pulse was $5.1 \mu \mathrm{s}$. The number of transitions for each time $t_{1}$ was 16 (the initial $t_{1}$ value was $250 \mu \mathrm{s}$ ). The repetition rate was ten seconds. (A $\mathrm{T}_{1}$-measurement inversion recovery $\}$ yielded $\mathrm{T}_{1}\left(\mathrm{I}_{1}\right)=2.3, \mathrm{~T}_{1}\left(\mathrm{I}_{2}\right)=2.3$ and $\left.\mathrm{T}_{1}\left(\mathrm{I}_{3}\right)=2.1 \mathrm{~s}\right)$. The total acquisition time was $9.7 \mathrm{~h}$ and the computing time was about $1.5 \mathrm{~h}$. Before carrying out the Fourier transformation in the $t_{2}$ and $t_{1}$ dimension each FID was multiplied by a certain window function. In the $t_{2}$ dimension the window function $\exp (2 \mathrm{t} / \mathrm{AQT}) \cdot \cos (2 \pi \mathrm{t} / \mathrm{AQT})$, and in the $t_{1}$ dimension the window function $\sin (\pi \mathrm{t} / \mathrm{AQT})(\mathrm{AQT}=$ acquisition time) were used. Both window functions improve the resolution and, in addition, the second filter function supresses strong tails and broad peaks (e.g., the signal of proton 3 ). All $2 \mathrm{D}-\mathrm{J}$ spectra are absolute value spectra.

\section{Results}

When NMR is used for analysis of diastereoisomers the corresponding multiplets may lie very close together even at high fields. The application of $2 \mathrm{D}-\mathrm{J}$ spectroscopy for the identification of diastereoisomers will be demonstrated by taking the molecule (1) as a representative example. In (1)

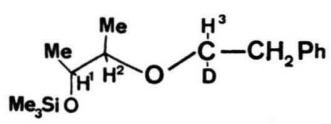

there are three asymmetric carbon atoms, thus four diastereomers should be discernable by NMR; however, by ${ }^{13} \mathrm{C}-\mathrm{NMR}(25.2 \mathrm{MHz})$ only one and by ${ }^{2} \mathrm{H}-\mathrm{NMR}(61.4 \mathrm{MHz})$ only two isomers could be detected. The $400 \mathrm{MHz}{ }^{1} \mathrm{H}-\mathrm{NMR}$ spectrum of (1) dissolved in $\mathrm{CDCl}_{3}$ is also complex (cf. Fig. la): there is one major isomer I and its signals for the protons 1,2 , and 3 at $\delta\left(\mathrm{I}_{1}\right)=3.78, \delta\left(\mathrm{I}_{2}\right)=3.29$, and $\delta\left(\mathrm{I}_{3}\right)=3.65$ can be easily identified as two doublets of quartets, and a "triplet of triplets", respectively. The latter splitting results from the coupling of 3 with the diastereotopic benzyl protons and with deuterium. The deuterium coupling should give a triplet splitting with the intensity ratio $1: 1: 1$; however, the $\mathrm{H}, \mathrm{D}$ coupling is small and partly hidden in the line width, therefore a deceiving $1: 2: 1$ intensity ratio is observed for each component of $\mathrm{I}_{3}$. The other signals cannot be assigned directly, in particular the resonances around $\delta=3.55$ are unresolved. The assignment becomes clear from the $400 \mathrm{MHz}$ 2D-J spectrum of (1) (cf. Fig. $1 \mathrm{~b}$ and Fig. 2). For clarity and experimental reasons (cf. experimental

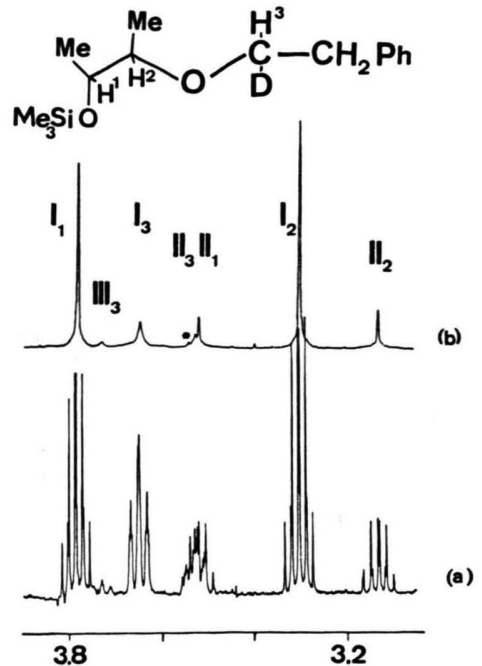

Fig. 1. (a) Part of the one-dimensional $400 \mathrm{MHz}$ ${ }^{1} \mathrm{H}-\mathrm{NMR}$ spectrum of (1), (b) Projection of the corresponding part of the $2 \mathrm{D}-\mathrm{J}$-resolved spectrum of (1) in the $\omega_{1}$ direction on the $\omega_{2}$ axis. The singlets $I_{1}, I_{2}, I_{3}$, $\mathrm{II}_{1}, \mathrm{II}_{2}, \mathrm{II}_{3}$, and $\mathrm{III}_{3}$ are clearly resolved and yield the chemical shifts (* denotes an impurity). The deuterium coupling on $\mathrm{I}_{3}, \mathrm{II}_{3}$, and $\mathrm{III}_{3}$ can be detected in an expansion of these signals (cf. also Fig. 2 and Fig. 4).

part) only the signals of the protons 1,2 , and 3 are presented. The major isomer I can be easily identified by its signals for the protons 1,2 and 3 , and by the splittings of these resonances. Two further stereoisomers II and III can be detected in the twodimensional spectrum of (1) (cf. the assignment in Fig. 2). They are most easily identified in the projection of the 2D-J spectrum (cf. Fig. $1 \mathrm{~b}$ ). In contrast to the one-dimensional $400 \mathrm{MHz}{ }^{1} \mathrm{H}-\mathrm{NMR}$

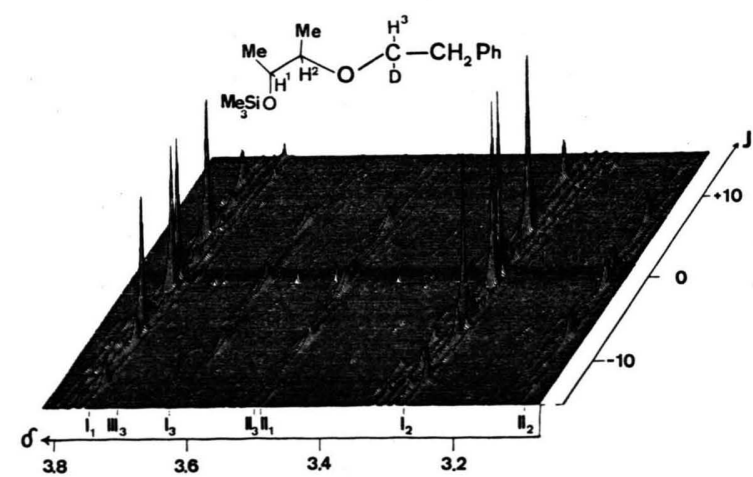

Fig. 2. $400 \mathrm{MHz} 2 \mathrm{D}-\mathrm{J}$ spectrum of (1). Only the resonances 1,2 , and 3 have been recorded. In the $\omega_{2}$ dimension $(\delta=3.1-3.9)$ the chemical shifts of the isomers I ( $82 \%)$, II ( $15 \%)$, and III ( $3 \%$ ) are presented whereas in the $\omega_{1}$ dimension the homonuclear coupling constants are displayed. 


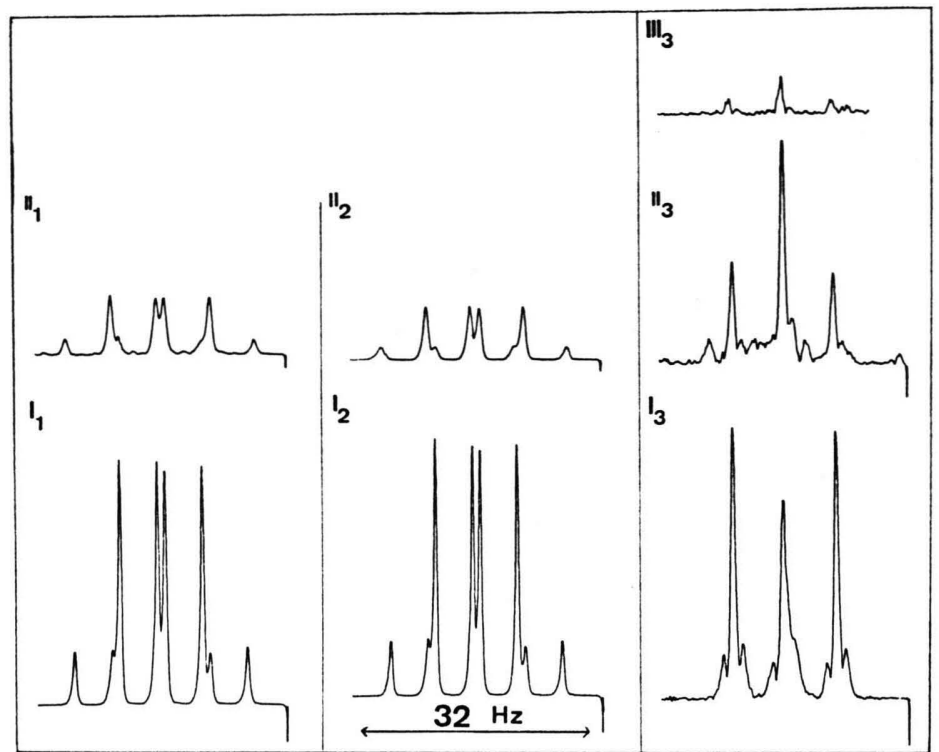

Fig. 3. Multiplets of the signals $\mathrm{I}_{1}-\mathrm{III}_{3}$ resulting from the projection in the $\omega_{2}$ direction of the 2D-J spectrum of Fig. 2. The humps in the signals of proton 3 stem from its deuterium splitting. spectrum the resonances of the protons $\mathrm{II}_{1}$ and $\mathrm{II}_{3}$ are now clearly separated, and one signal is recognized as an impurity (marked with *). Furthermore, a signal $\mathrm{III}_{3}$ of the third stereoisomer can be detected at $\delta\left(\mathrm{II}_{3}\right)=3.76$. These assignments are confirmed by the homonuclear coupling patterns of the seven signals $\left(\mathrm{I}_{1}, \mathrm{I}_{2}, \mathrm{I}_{3}, \mathrm{II}_{1}, \mathrm{II}_{2}, \mathrm{II}_{3}\right.$, and $\left.\mathrm{III}_{3}\right)$. The multiplets can be obtained from the two dimensional plot (cf. Fig. 2) by projection in the $\omega_{2}(\delta)$ direction on the $\omega_{1}(J)$ axis. These projections are shown in Fig. 3.

The line width (homonuclear couplings) is controlled by spin-spin relaxation rather than by magnetic field inhomogenity. Therefore homonuclear coupling constants which are obtained from 2D-J spectra are of high accuracy, as long as the magnetic field remains constant with time.

The relative intensity of each of the resonances 1,2 , and 3 should be one. The deviation from this ratio (cf. Fig. 1 and Fig. 2) results from apodisation of the FID in $t_{1}$ and $t_{2}$ dimension with certain window functions ( $c f$. experimental part). In particular the apodisation which was chosen in the $t_{2}$ dimension suppresses broad peaks and therefore the intensity of the resonances of proton 3 (with partly unresolved deuterium coupling in the $\omega_{2}$ dimension) is reduced. However, when the intensity of corresponding multiplets is compared (i.e. $\mathrm{I}_{3}: \mathrm{II}_{3}: \mathrm{III}_{3}$ ), the real ratio of the stereoisomers ( $\mathrm{I}(82 \%)$, II
$(15 \%)$, and III $(3 \%))$ should be obtained. The assignment of the stereoisomers I-III is based on their NMR data and on chemical arguments. In I (the major stereoisomer) the relative configuration of the methyl groups is believed to be threo $\left(\mathrm{R}^{*} \mathrm{R}^{*}\right)$ and in II erythro $\left(\mathrm{R}^{*} \mathrm{~S}^{*}\right)$, since in I the coupling of the protons 1 and 2 is $3 J_{1,2}(\mathrm{I})=5.15 \mathrm{~Hz}$ and in II $3 J_{1,2}(\mathrm{II})=7.24 \mathrm{~Hz}$. The minor stereoisomer (III) must therefore have the relative configuration of the methyl groups identical to either that of I or that of II (having only $\mathrm{H}$ and $\mathrm{D}$ of the CHD group interchanged). No signals for the protons III $_{1}$ and $\mathrm{III}_{2}$ are observed, since they must be virtually degenerate with the corresponding signals of either I or II. Precise integration showed that $\mathrm{III}_{1}$ and $\mathrm{III}_{2}$ coincide with the corresponding signals of $\mathrm{I}$, thus establishing the relative configuration of the methyl groups of III as threo. This interpretation agrees well to the chemistry of (1) [10]: (1) was prepared from the corresponding 2-benzyl-4,5-dimethyldioxolane which consisted of $85 \%$ of racemic isomer (threo) and of $15 \%$ of meso isomer (erythro).

As pointed out earlier the homonuclear couplings exclusively are displayed in the $\omega_{1}(J)$ frequency dimension whereas heterocouplings in general are displayed in the $\omega_{2}(\delta)$ dimension. This results from the fact that only the transitions of the proton spin system are affected by the refocusing $180^{\circ}$ pulse. In the case of a homonuclear $\mathrm{AX}$ spin system the $\mathrm{A}_{1}$ 
and $A_{2}$, as well as $X_{1}$ and $X_{2}$ transitions are changed, while in a heteronuclear $\mathrm{AX}$ spin system $\left(\mathrm{A}={ }^{1} \mathrm{H}\right.$ and $\mathrm{X}={ }^{2} \mathrm{H}$ ) only the $\mathrm{A}_{1}$ and $\mathrm{A}_{2}$ transitions are interchanged by the $180^{\circ}$ pulse. In (1) the splitting of proton 3 should be a triplet of a triplet, due to coupling with the diastereotopic benzyl protons and with deuterium. As illustrated in Fig. 2 and more clearly in an expansion (cf. Fig. 4) the homonuclear
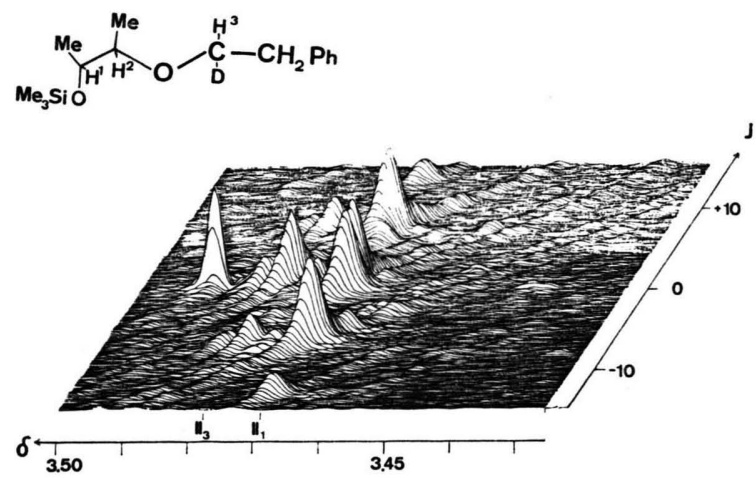

Fig. 4. Expansion ( $\delta=3.43-3.50)$ of the 2D-J-resolved spectrum of Fig. 2. The homonuclear couplings of $\mathrm{II}_{1}$ and $\mathrm{II}_{3}$ are displayed in the $\omega_{1}(J)$ dimension, whilst the coupling of $\mathrm{II}_{3}$ with deuterium is displayed in the $\omega_{2}(\delta)$ dimension. The singlet at $\delta=3.49$ originates from an impurity * (cf. Fig. $1 \mathrm{~b}$ ).

couplings of $\mathrm{II}_{3}$ are displayed in the $\omega_{1}(\mathrm{~J})$ dimension whereas the smaller triplet splitting of $\mathrm{II}_{3}$ due to deuterium is conserved in the $\omega_{2}(\delta)$ direction. Thus 2D-J NMR can be used as a method for identification of heterocouplings especially in those cases where the heteroresonance frequency is unknown or where decoupling experiments cannot be performed. (For instance it is not possible to observe protons and to decouple deuterium while locked on deuterium. Experiments in which ${ }^{13} \mathrm{C}$ is observed and ${ }^{31} \mathrm{P}$ is decoupled while locked on deuterium can also not be carried out since the frequency differ- ence of ${ }^{31} \mathrm{P}$ and ${ }^{2} \mathrm{H}$ comes close to the ${ }^{13} \mathrm{C}$ resonance frequency.)

\section{Discussion}

In many cases it is possible to record NMR spectra over of a small spectral range of interest (with the help of suitable filters folded frequencies can be largely suppressed. Consequently $2 \mathrm{D}-\mathrm{J}$ spectra can be obtained with high digital resolution in $\omega_{1}$ and $\omega_{2}$ directions (a typical data matrix consists of $4096 \times 128$ data points) and within reasonable recording and computing time (typically in overnight runs). Of special value is the projection of a $2 \mathrm{D}-\mathrm{J}$ spectrum in the $\omega_{1}(\mathrm{~J})$ direction: it results in a type of broadband proton decoupled one dimensional proton spectrum with true intensity ratios of the singlets (if the appropriate window functions for the apodisation of the FID are used). From the resulting spectrum of singlets symmetry arguments for structure elucidation as well as frequencies for homonuclear decoupling experiments can be obtained. Furthermore mixtures of different compounds or isomers can be easily investigated by means of 2D-J NMR (even at moderate field strength) as long as each isomer has a spectrum which is nearly first order. In particular stereoisomers can be easily detected by 2D-J NMR spectroscopy. Other techniques for identifying stereoisomers such as labeling with deuterium and studying the deuterium resonances may be unfavourable since the high frequency dispersion of the protons is reduced in the deuterium spectrum. The use of shift reagents can also become difficult since the lines broaden (especially in the case of high field spectrometers) and stereoisomers of low intensity are difficult to detect. Hetero-couplings can be readily detected with the help of $2 \mathrm{D}-\mathrm{J}$ NMR, when only the multiplets of interest are recorded.
[1] J. Jeener, Ampere Int. Summer School II, Bako Polje, Yugoslavia 1971.

[2] P. C. Lauterbur, Nature (London) 242, 190 (1973).

[3] A. Kumar, D. Welti, and R. R. Ernst, J. Magn. Res. 18, 69 (1975).

[4] W. P. Aue, E. Bartholdi, and R. R. Ernst, J. Chem. Phys. 64, 2229 (1976).

[5] G. Bodenhausen, R. Freeman, R. Niedermeyer, and D. L. Turner, J. Magn. Reson. 26, 133 (1977).

[6] A. Wokaun and R. R. Ernst, Mol. Phys. 36, 317 (1978).
[7] R. Freeman,

a) Abstr. ISMAR Ampere Internat. Conf. Magn. Res., Delft, Holland 1980;

b) A. Bax, R. Freeman, and S. P. Kampsell, J. Am. Chem. Soc. 102, 4849 (1980).

[8] S. Macura, Y. Huang, and R. R. Ernst, Abstr. ISMAR Ampere Internat. Conf. Magn. Res., Delft, Holland 1980.

[9] E. L. Hahn and D. E. Maxwell, Phys. Rev. 88, 1070 (1952).

[10] W. J. Richter, to be published. 